\title{
Observational study for the assessment of platelet function in anesthetized patients (AGREE Study)
}

Llau JV, Izquierdo A, Del Río E, Garzando M, Ferrandis R

Hospital Clinic Universitari of Valencia, Dept of Anaesthesiology \& Intensive Care,Valencia, Spain

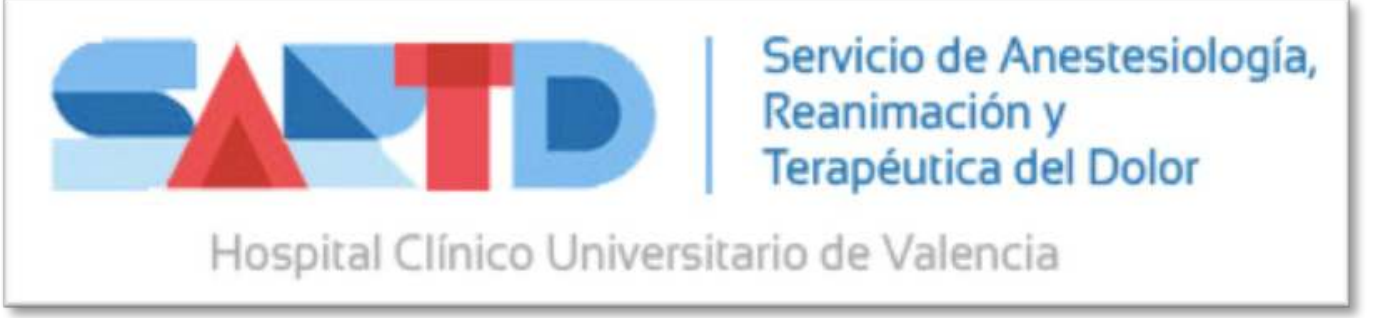

\section{Background and Goal of Study}

Many surgical patients regularly receive non-steroidal anti-inflammatory drugs (NSAIDs) for postoperative pain treatment. Its action mechanisms include COX-I inhibition, similar to the antiplatelet action of the aspirin.

The goal of AGREE is to determine the antiaggregant effect of NSAIDs given at the end of surgery in order to prevent postoperative pain

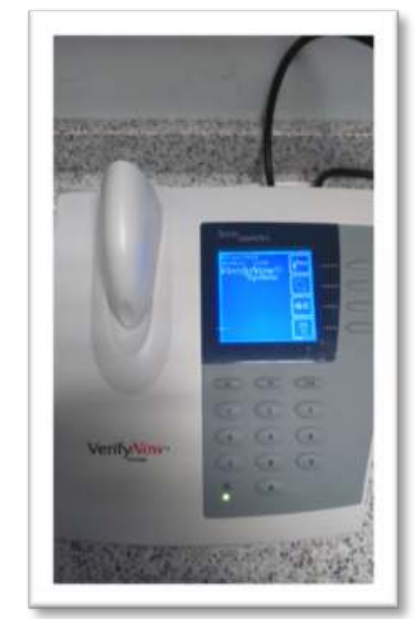

VerifyNow device

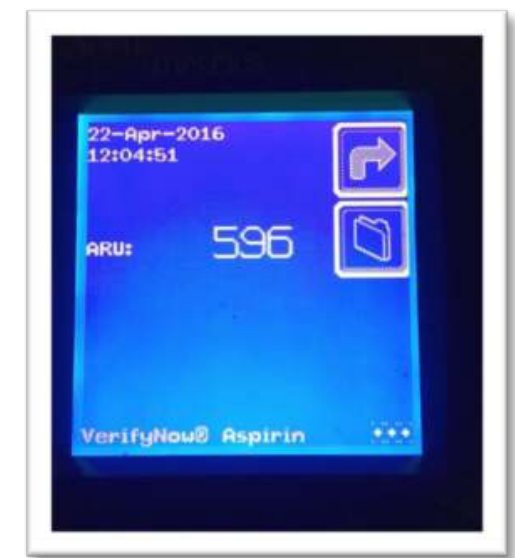

Result of $>550$ ARU

is consistent with antiaggregation

\section{Materials and methods}

AGREE is a pilot, prospective, observational study, approved by the hospital ethical committee. A group of surgical patients over 18 years, not having received any medication that could have disturbed haemostasis within seven days before the intervention were included after obtaining informed consent.

Acetaminophen ( $\mathrm{lg}$ ) and Dexketoprophen $(50 \mathrm{mg})$ was administered intravenously to every patient $30-45^{\prime}$ before the end of the surgery.

Platelet function was tested with the aspirin aggregation test using the point- of-care test VerifyNow

(Accumetrics, Inc., San Diego, CA-USA).

Results are expressed in ARU (Aspirin Reactive Units): $>550$ ARU is consistent with normal platelet reactivity without dysfunction related with aspirin, and

$<550$ ARU is consistent with platelet dysfunction related with aspirin.

Two blood samples were obtained:

(TI) $30^{\prime}\left(+/-10^{\prime}\right)$ before the administration of the analgesia protocol;

(T2) $20^{\prime}(+/-10)$ after the administration ended.

\section{Results and discussion}

12 patients were included (3/9 male/female, 10/2 ASAll/III), median age 57 years (range 39-77).

I I/I 2 presented a normal platelet reactivity result before the administration of the analgesia protocol ( $\mathrm{TI}$ ). From those II patients with >550 ARU, 10 changed the profile, showing platelet dysfunction after the NSAID infusion (T2) (see table).

TABLE

\begin{tabular}{|c|c|c|c|c|c|c|c|c|c|c|c|c|c|}
\hline & PATIENT & 1 & 2 & 3 & 4 & 5 & 6 & 7 & 8 & 9 & 10 & 11 & 12 \\
\hline T1 & ARU Pre-NSAID & $552^{m !}$ & $556^{\prime 7}$ & $577^{5}$ & $621^{19}$ & $650^{13}$ & $543^{\text {का }}$ & $601^{12}$ & $620^{19}$ & $601^{\mathrm{No}}$ & $652^{27}$ & $636^{25}$ & $606^{17}$ \\
\hline T2 & ARU Post-NSAID & $386^{(x)}$ & $589^{m}$ & $537^{(0)}$ & $409^{(0)}$ & $387^{(\alpha)}$ & $466^{(2)}$ & $523^{\text {ण }}$ & $425^{\text {एग }}$ & $523^{(0)}$ & $512^{\text {मा }}$ & $407^{101}$ & $386^{(\pi / 4}$ \\
\hline
\end{tabular}

$\left(^{*}\right)=$ no platelet dysfunction $(\#)=$ platelet dysfunction

\section{Conclusion(s)}

In most cases NSAID administration as analgesia protocol produces platelet impairment similar to the one produced by aspirin. Although further studies are required to determine if NSAID administration could increase postoperative bleeding, in weak and elderly patients caution should be advised. 